\title{
ANOMALOUS MAGNETORESISTANCE IN MULTI-LEVEL QUANTUM WELLS
}

\author{
N.S. Averkiev, L.E. Golub and G.E. Pikus \\ A.F. Ioffe Physico-Technical Institute \\ Politechnicheskaya 26, 194021 St. Petersburg, Russia
}

\begin{abstract}
Effect of intensive interlevel transitions in quantum well and strong spin-orbit interaction on weak localization is considered. Anomalous magnetoresistance in classically weak fields is calculated for $p$-type quantum wells based on $A_{3} B_{5}$ semiconductors. It is shown that the sign of magnetoresistance changes with varying doping level and the role of the intersubband transitions in weak localization effects depends dramatically on a view of the scattering potential.
\end{abstract}

PACS numbers: 71.55.Eq, 71.70.Ej, 73.20.Fz

\section{Introduction}

The anomalous magnetoresistance in weak fields is well known to be caused by weak localization. This phenomenon results from the interference of waves propagating through the closed trajectory in the opposite directions. The interference is destroyed by a magnetic field and the anomalous contribution to the conductivity decreases.

Beside the magnetic field the anomalous contribution changes due to the phase and spin relaxation processes. In the previous theories [1-5] they were assumed slower than momentum relaxation processes. But in the intensively investigated $p$-type heterostructures there is the strong spin-orbit interaction and therefore the spin relaxation time is of the same order as the momentum relaxation one.

Moreover, in the real heterostructures a few size-quantized levels can be filled. The transitions between them can also destroy the interference and change the anomalous contribution to the magnetoresistance. In the $p$-type quantum wells (QWs) the rate of these transitions can vary with subband filling and QW width.

The aim of this work is to create a weak localization theory in the case of strong spin-orbit interaction taking into account filling of a few subbands of size quantization. 


\section{Equation for Cooperon}

The anomalous contribution to the conductivity caused by the weak localization effects can be presented as follows:

$$
\Delta \sigma=\frac{e^{2}}{\pi \hbar} \int \frac{\mathrm{d}^{2} q}{(2 \pi)^{2}} A(q),
$$

where $A(q)$ looks like a sum of diffusion poles. To calculate the magnetoconductivity in classically weak fields one has to replace $[1,2]$

$$
\int \frac{\mathrm{d}^{2} q}{(2 \pi)^{2}} \rightarrow \frac{e H}{4 \pi^{2} \hbar c} \sum_{n}, \quad q^{2} \rightarrow \frac{e H}{\pi \hbar c}(n+1 / 2),
$$

where $H$ is the projection of the magnetic field on the normal to the QW plane.

To obtain the quantity $A(q)$ one has to find the intensity of the interference of waves propagating in the opposite directions (Cooperon). Since every electronic wave depends on both the spin state and the subband number, the Cooperon is the matrix in respect of these subscripts. The equation for it is the following:

$$
\begin{aligned}
& \mathcal{C}_{\gamma \delta}^{\alpha \beta}\left(k, k^{\prime}, q\right)=\left\langle V_{\alpha \beta}\left(-k,-k^{\prime}\right) V_{\gamma \delta}\left(k+q, k^{\prime}+q\right)\right\rangle \\
& \quad+\sum_{\mu \nu} \int \frac{\mathrm{d}^{2} g}{(2 \pi)^{2}}\left\langle V_{\alpha \mu}(-k,-g) V_{\gamma \nu}(k+q, g+q)\right\rangle \\
& \quad \times \mathcal{C}_{\nu \delta}^{\mu \beta}\left(g, k^{\prime}, q\right) G_{\nu}^{R}(g+q) G_{\mu}^{A}(-g),
\end{aligned}
$$

where $-k$ and $-k^{\prime}$ are the initial and final momenta of the one of the scattering waves and $\boldsymbol{q}$ is the summary momentum of both scattering waves. The Greek subscripts enumerate the spin states in different subbands of size quantization. Advanced and retarded Green's functions, $G_{\nu}^{\mathrm{A}, \mathrm{R}}(k)$, are chosen in the form given in Ref. [6]. By $\left\langle V_{\alpha \beta} V_{\gamma \delta}\right\rangle$ we understand biline combinations of scattering potential Fourier-images averaged over the scatter positions. As a result of this average only the following combinations are nonzero in a symmetrical $\mathrm{QW}$ : $\left\langle V_{11} V_{11}\right\rangle,\left\langle V_{11} V_{22}\right\rangle,\left\langle V_{12} V_{21}\right\rangle$, where the subscripts "1" and "2" enumerate only the pairs of spin states in all subbands.

Here we use the so-called spherical approximation for the description of the valence band states in QW. According to this model the energies, $E_{\alpha}$, do not depend on the vector $k$ directions and $V_{\alpha \nu}\left(k, k^{\prime}\right)$ in Eq. (2) depends on $\left|k-k^{\prime}\right|$. Therefore the total relaxation times of the states $|\alpha k\rangle, \tau_{\alpha}$, are constants. Analogously, the phenomenologically introduced phase relaxation times, $\tau_{\varphi}^{(\alpha)}$, will be also assumed $k$-independent.

Equation (2) is known to have the peculiarity at $\boldsymbol{q} \rightarrow 0$. Below this equation will be considered for the case of filling of two subbands.

When a few subbands are filled the intersubband transitions appear which act as a new phase breaking mechanism. Its characteristic feature from the other is that the states in different subbands cannot interfere and contribute to the Cooperon because they have different momenta at the Fermi surface.

For the sake of definiteness we consider the situation when in QW two subbands of size quantization are filled: the lower $(l)$ and the upper $(u)$. Thus we have to consider the four states: $l 1, l 2$ belong to the $l$-subband and $u 1, u 2$ are in 
the $u$-subband. We choose these degenerate states in each subband so that they transform one to another under the time inversion operation.

In this case in $p$-QWs the spin relaxation in the $l$-subband is fast and therefore only the two-particle states with zero total momentum give contribution to the weak localization. For this reason the Cooperon can be represented in the following form:

$$
\begin{aligned}
& \mathcal{C}_{\gamma \delta}^{\alpha \beta}(q)=s_{l}(q) \mathcal{S}_{l \gamma}^{\alpha} \mathcal{S}_{l \delta}^{\beta}+s_{u}(q) \mathcal{S}_{u \gamma}^{\alpha} \mathcal{S}_{u \delta}^{\beta}+s_{l u}(q) \mathcal{S}_{l \gamma}^{\alpha} \mathcal{S}_{u \delta}^{\beta}+s_{u l}(q) \mathcal{S}_{u \gamma}^{\alpha} \mathcal{S}_{l \delta}^{\beta} \\
& +p_{u 1}(q)\left(\mathcal{P}_{u+\gamma}^{\alpha} \mathcal{P}_{u+\delta}^{\beta}+\mathcal{P}_{u-\gamma}^{\alpha} \mathcal{P}_{u-\delta}^{\beta}\right)+p_{u 0}(q) \mathcal{P}_{u 0}{ }_{\gamma}^{\alpha} \mathcal{P}_{u 0 \delta}^{\beta} \text {. }
\end{aligned}
$$

Here the non-zero components of $\mathcal{S}_{l \gamma}^{\alpha}, \mathcal{S}_{u \gamma}^{\alpha}$ and $\mathcal{P}_{u i_{\gamma}}^{\alpha}$ are: $\mathcal{S}_{l l 2}^{l i}=-\mathcal{S}_{l 1}^{l 2}=\mathcal{S}_{u u_{2}}^{u 1}=$ $-\mathcal{S}_{u u 1}^{u 2}=\mathcal{P}_{u 0 u 2}^{u 1}=1 / \sqrt{2}, \mathcal{P}_{u+u 1}^{u 1}=\mathcal{P}_{u-u 2}^{u 2}=1$.

\section{Magnetoconductivity calculation}

Since the elastic scattering from one subband to another is accompanied by a large change of quasimomentum, the method of the calculation and the view of the functions $s_{i}$ and $p_{i}$ in Eq. (3) depend on the properties of the scattering potential.

If it is short-range then the intersubband transition times, $\tau_{l u}$ and $\tau_{u l}$, are comparable with the momentum relaxation times in each subband, $\tau_{l}$ and $\tau_{u}$. The states with non-zero total momentum in the upper subband relax quickly due to often transitions to the lowest one. These states do not give a contribution to the weak localization effects.

The states with zero total momentum in both subbands mix significantly and their contribution is described by the averaged diffusion coefficient, $D$, and the phase breaking time, $\tau_{\varphi}$. Therewith the quantity $A(q)$ is positive and has the following form:

$$
A(q)=\frac{1}{q^{2}+\left(D \tau_{\varphi}\right)^{-1}}
$$

where

$$
\begin{gathered}
D=\frac{v_{\mathrm{F}}^{(l)^{2}} \tau_{l}}{2} \frac{N_{l}\left(1-N_{u} \tau_{u} U_{1}\right)}{Z}+\frac{v_{\mathrm{F}}^{(u)^{2}} \tau_{u}}{2} \frac{N_{u}\left(1-N_{l} \tau_{l} L_{1}\right)}{Z}+v_{\mathrm{F}}^{(l)} v_{\mathrm{F}}^{(u)} \tau_{l} \tau_{u} \frac{N_{l} N_{u} W_{1}}{Z}, \\
Z=\left[\left(1-N_{l} \tau_{l} L_{1}\right)\left(1-N_{u} \tau_{u} U_{1}\right)-N_{l} N_{u} \tau_{l} \tau_{u} W_{1}^{2}\right]\left(N_{l}+N_{u}\right), \\
\tau_{\varphi}^{-1}=\frac{N_{l} / \tau_{\varphi}^{(l)}+N_{u} / \tau_{\varphi}^{(u)}}{N_{l}+N_{u}} .
\end{gathered}
$$

In Eqs. (5), (6) $v_{\mathrm{F}}^{(\alpha)}$ and $N_{\alpha}$ are respectively the carrier velocity and the density of states at the Fermi surface in the subband $\alpha$, and $L_{1}, U_{1}, W_{1}$ represent first Fourier harmonics of the scattering potential

$$
\begin{aligned}
& L_{1}=\frac{2 \pi}{\hbar} \int \frac{\mathrm{d} \varphi}{2 \pi}\left\langle\left|V_{l 1, l 1}(\varphi)\right|^{2}+\left|V_{l 1, l 2}(\varphi)\right|^{2}\right\rangle \mathrm{e}^{-\mathrm{i} \varphi}, \\
& U_{1}=\frac{2 \pi}{\hbar} \int \frac{\mathrm{d} \varphi}{2 \pi}\left\langle\left|V_{u 1, u 1}(\varphi)\right|^{2}+\left|V_{u 1, u 2}(\varphi)\right|^{2}\right\rangle \mathrm{e}^{-\mathrm{i} \varphi},
\end{aligned}
$$




$$
W_{1}=\frac{2 \pi}{\hbar} \int \frac{\mathrm{d} \varphi}{2 \pi}\left\langle\left|V_{l 1, u 1}(\varphi)\right|^{2}+\left|V_{l 1, u 2}(\varphi)\right|^{2}\right\rangle \mathrm{e}^{-\mathrm{i} \varphi} .
$$

From Eq. (4) it follows that in the case of the short-range potential the magnetoconductivity is negative and it is described by a single characteristic magnetic field

$$
\delta \sigma(H)=-\frac{e^{2}}{4 \pi^{2} \hbar} f_{2}\left(\frac{H}{H_{\varphi}}\right),
$$

where $H_{\varphi}=\hbar c /\left(4 e D \tau_{\varphi}\right)$. Here $f_{2}$ is the standard function: $f_{2}(x)=\ln x+$ $\psi(1 / 2+1 / x)$, where $\psi(y)$ is Digamma-function.

If the scattering potential is smooth then the intersubband transitions are suppressed and their characteristic times exceed the momentum relaxation times. However, the relationship between $\tau_{l u}, \tau_{u l}$ and $\tau_{\varphi}^{(l)}, \tau_{\varphi}^{(u)}$ can be arbitrary.

The expressions for $s_{i}$ and $p_{i}$ are cumbersome in this case but the magnetoconductivity has the more simple view

$\delta \sigma(H)=\frac{e^{2}}{4 \pi^{2} \hbar}\left[2 f_{2}\left(\frac{H}{H_{\|}^{(u)}}\right)+f_{2}\left(\frac{H}{H_{\perp}^{(u)}}\right)-f_{2}\left(\frac{H}{H_{-}}\right)-f_{2}\left(\frac{H}{H_{+}}\right)\right]$,

where the characteristic magnetic fields are

$$
H_{\|, \perp}^{(u)}=\frac{\hbar c}{4 e D_{u}^{(0)}}\left(\frac{1}{\tau_{\varphi}^{(u)}}+\frac{1}{\tau_{\|, \perp}^{(u)}}+\frac{1}{\tau_{u l}}\right), \quad H_{ \pm}=\frac{\hbar c}{4 e}\left(A_{l}+A_{u} \pm F\right) .
$$

Here

$$
\begin{aligned}
& A_{l}=\frac{1}{2 D_{l}^{(0)}}\left[\frac{1}{\tau_{\varphi}^{(l)}}+\frac{1}{\tau_{l u}}\right], \quad A_{u}=\frac{1}{2 D_{u}^{(0)}}\left[\frac{1}{\tau_{\varphi}^{(u)}}+\frac{1}{\tau_{u l}}\right], \\
& F=\left[\left(A_{l}-A_{u}\right)^{2}+\frac{1}{D_{l}^{(0)} \tau_{l u} D_{u}^{(0)} \tau_{u l}}\right]^{1 / 2} .
\end{aligned}
$$

The subscript "0" means that the respective quantities have to be calculated without regard for the intersubband transitions. The times $\tau_{\|}^{(u)}$ and $\tau_{\perp}^{(u)}$ describe the spin relaxation in the upper subband.

At $H_{ \pm}<H_{\|, \perp}^{(u)}$ the curve $\delta \sigma(H)$ changes its sign, and if $H_{ \pm}>H_{\|, \perp}^{(u)}$ then $\delta \sigma(H)$ is positive everywhere over the validity region of the diffusion theory considered here.

The spin relaxation times in the upper subband depend strongly on its filling, i.e., on the parameter $\left(E_{\mathrm{F}}-\Delta\right) / \Delta$. Here $E_{\mathrm{F}}$ is the Fermi energy and $\Delta$ is the minimal energy distance between the first and the second hole subbands. If this ratio is small then the times $\tau_{\|, \perp}^{(u)}$ far exceed the momentum relaxation time. If, otherwise, $\left(E_{\mathrm{F}}-\Delta\right) / \Delta \approx 1$ then the mixing of the heavy- and light-hole states is significant even in the excited subband and the spin relaxation times in it, $\tau_{\|, \perp}^{(u)}$, are comparable with the momentum relaxation time $\tau_{u}$. It means that with increasing carrier concentration in the upper subband the characteristic magnetic fields, $H_{\|}^{(u)}$ 
and $H_{\perp}^{(u)}$, became of the same order as $H_{\mathrm{tr}}=\hbar c /(4 e D \tau) \gg H_{\varphi}$. While doing this the first two terms in Eq. (11) disappear.

The expression for the conductivity (11) is valid at the arbitrary relationship between the intersubband transition times and the wave function phase breaking times. In the limiting cases the expressions for $H_{ \pm}$are simplified. If the potential is so smooth that $\tau_{l u} \gg \tau_{\varphi}^{(l)}$ and $\tau_{u l} \gg \tau_{\varphi}^{(u)}$ then

$$
H_{-}=\frac{\hbar c}{4 e D_{l}^{(0)} \tau_{\varphi}^{(l)}}, \quad H_{+}=\frac{\hbar c}{4 e D_{u}^{(0)} \tau_{\varphi}^{(u)}} .
$$

It means that both subbands give independent contributions to the conductivity. In the opposite limit, $\tau_{l u} \ll \tau_{\varphi}^{(l)}, \tau_{u l} \ll \tau_{\varphi}^{(u)}$,

$$
H_{-}=\frac{\hbar c}{4 e D_{0} \tau_{\varphi}}, \quad H_{+}=\frac{\hbar c}{4 e}\left(\frac{1}{D_{l}^{(0)} \tau_{l u}}+\frac{1}{D_{u}^{(0)} \tau_{u l}}\right), \quad H_{+} \gg H_{-},
$$

where $\tau_{\varphi}$ coincides with (6) and the averaged diffusion coefficient $D_{0}(5)$ at rare intersubband transitions $\left(\left|W_{1}\right| \ll\left|L_{1}\right|,\left|U_{1}\right|\right)$ is

$$
D_{0}=\frac{N_{l} D_{l}^{(0)}+N_{u} D_{u}^{(0)}}{N_{l}+N_{u}}
$$

The formulae (15) are simple in the physical meaning. In the fields $H \sim$ $H_{-} \ll H_{+}$the conductivity has the standard form (10) which contains the averaged over both subbands quantities $D_{0}$ and $\tau_{\varphi}$. The second peculiarity appears at $H \geq H_{+} \gg H_{-}$because the phase breaking rate due to magnetic field became larger than the intersubband transition rate. It should be noted that both the peculiarities can be observed experimentally at the following relationship between the times

$$
\tau_{l, u} \ll \tau_{l u, u l} \ll \tau_{\varphi}^{(l, u)} .
$$

The second peculiarity is absent in Eq. (10) because $H_{+}$is about $H_{\text {tr }}$ at the scattering on the short-range potential and this value represents the limiting field for the diffusion theory.

\section{Conclusion}

The weak localization theory has been developed for QWs with a few filled subbands of size quantization. The expressions for the magnetoconductivity have been obtained at different relationships between the times of spin relaxation, momentum relaxation, phase relaxation and intersubband transitions. The characteristic magnetic fields have been calculated allowing for the real band structure of size-quantized systems.

\section{Acknowledgments}

This work has been partly supported by RFBR (grants 96-15-96955 and 98-02-18424), program "Physics of Solid State Nanostructures" (grant 97-1035) and Volkswagen Foundation. 


\section{R.eferences}

[1] S. Hikami, A. Larkin, Y. Nagaoka, Prog. Theor. Phys. 63, 707 (1980).

[2] B.L. Al'tshuler, A.G. Aronov, A.I. Larkin, D.E. Khmel'nitskii, Zh. Eksp. Teor. Fiz. 81, 768 (1981) [Sov. Phys. JETP 54, 411 (1981)].

[3] S.V. Iordanskii, Yu.B. Lyanda-Geller, G.E. Pikus, Pis'ma Zh. Eksp. Teor. 60, 199 (1994) [JETP Lett. 60, 206 (1994)].

[4] F.G. Pikus, G.E. Pikus, Phys. Rev. B 51, 16928 (1995).

[5] W. Knap, C. Skierbiszewski, A. Zduniak, E. Litwin-Staszewska, D. Bertho, F. Kobbi, J.L. Robert, G.E. Pikus, F.G. Pikus, S.V. Iordanskii, V. Moser, K. Zekenes, Yu.B. Lyanda-Geller, Phys. Rev. B 53, 3912 (1996).

[6] N:S. Averkiev, L.E. Golub, G.E. Pikus, Zh. Eksp. Teor. Fiz. 113, 1429 (1998). 